\title{
Inner-Truncated Disks - Are They Tied to Bars?
}

\author{
W. E. Baggett \\ Computer Sciences Corporation Astronomy Programs, STScI, \\ Baltimore, $M D 21218$
}

S. M. Baggett

STScI, 3700 San Martin Dr., Baltimore, MD 21218

K. S. J. Anderson

Dept. of Astron., New Mexico State Univ., Las Cruces, NM 88003

\section{Introduction}

An inner-truncated disk (ITD) brightness profile is one in which the inward extrapolation of the outer disk surface brightness significantly exceeds the observed surface brightness in a region near the galaxy center. Freeman (1970) identified these profiles as Type II profiles. Fourteen of Freeman's (1970) sample of 36 external galaxies are designated as Type II (ITD); seven of the ITD's are classified as barred galaxies and ITD's are found at all morphological types.

Ohta, Hamabe, \& Wakamatsu (1990) report that luminosity profiles perpendicular to the bars of six early-type galaxies all exhibit the Freeman Type II shape, while the feature is lost when azimuthally averaged profiles of the same galaxies are studied. They postulate that the bar formation resulted in an azimuthal redistribution of the stars, but no net radial redistribution.

Baggett, Baggett, \& Anderson (1992) found that approximately $25 \%$ of an inhomogeneous sample of 167 spiral galaxy brightness profiles were innertruncated and that barred spirals were slightly more likely to be ITD's than non-barred spirals. There was otherwise no clear trend with morphological type. Baggett et al. (1993) reported on a subset of the data used in the current study and confirmed the $25 \%$ global rate of occurrence. They found a weak tendency for early-type spirals to have a higher rate of occurrence of ITD's, and confirmed that barred spirals are more likely to be ITD's than non-barred spirals.

\section{Data and Profile Fitting}

The sample we are using consists of photographic $V$-band major axis brightness profiles of 659 spiral and lenticular galaxies from the Photographic Atlas of Northern Bright Galaxies (Kodaira et al. 1990; hereafter, PANBG). We have chosen galaxies from the PANBG sample by selecting all objects which are classified as lenticular or spiral in the Third Reference Catalogue of Bright Galaxies (de Vaucouleurs et al. 1991; hereafter RC3) and which have an inclination of less 
than 60 degrees to our line of sight. We have successfully performed bulge-disk decomposition on 411 brightness profiles which meet these criteria.

We used the IRAF/STSDAS task "nfit1d" to perform bulge-disk decomposition on the brightness profiles of the selected galaxies. We utilized the de Vaucouleurs' law (de Vaucouleurs 1953) for the bulge component:

$$
I_{B}(r)=I_{e} \times 10^{-3.33\left[\left(r / r_{e}\right)^{0.25}-1\right]}
$$

and an inner-truncated exponential disk (Kormendy 1977) for the disk component:

$$
I_{D}(r)=I_{0} \times e^{\left[-\left(r / r_{0}\right)-\left(r_{h} / r\right)^{3}\right]} .
$$

\section{Quantitative Definition of ITD}

One of the problems with previous studies of ITD's (Freeman 1970; Jafarov and Zasov 1989; Baggett, Baggett, \& Anderson 1992) is the lack of an objective, quantitative measure of the truncation. We have addressed this shortcoming by computing the distance-independent ratio $r_{h} / r_{0}$, and counting the number of galaxies which have a value of the ratio larger than a certain value. It seems natural to limit the definition of ITD's to disk-related parameters, so Table 1 shows the number and percentage of galaxies of each bar class which meet the various minimum values of the $r_{h} / r_{0}$ ratio.

Table 1. Relative Hole Size Statistics

\begin{tabular}{rcccccccc}
\hline & \multicolumn{2}{c}{ Non-Barred (SA) } & \multicolumn{2}{c}{ Barred (SB) } & \multicolumn{2}{c}{ Mixed (SAB) } & \multicolumn{2}{c}{ All Bar Classes } \\
Ratio & Number & $\%$ & Number & $\%$ & Number & $\%$ & Number & $\%$ \\
\hline$r_{h} / r_{0} \geq 0.0$ & 24 & 18.5 & 50 & 41.3 & 39 & 27.3 & 116 & 28.2 \\
$r_{h} / r_{0} \geq 0.5$ & 23 & 17.7 & 50 & 41.3 & 38 & 26.6 & 114 & 27.7 \\
$r_{h} / r_{0} \geq 1.0$ & 21 & 16.2 & 46 & 38.0 & 28 & 19.6 & 98 & 23.8 \\
\hline \hline
\end{tabular}

The result for $r_{h} / r_{0} \geq 1.0$ is in line with the results of Baggett, Baggett, \& Anderson (1992), so we choose to define ITD's as galaxies which have $r_{h} / r_{0} \geq$ 1.0 .

Using this definition of ITD, we have investigated the frequency of occurrence as a function of morphological type and bar class; Table 2 summarizes these relationships.

Table 2. T-Type/Bar Class Distributions of ITD's

\begin{tabular}{ccccccccc}
\hline & All Bar Classes & \multicolumn{2}{c}{ Non-Barred } & \multicolumn{2}{c}{ Barred } & \multicolumn{3}{c}{ Mixed } \\
T-Type Range & $N_{I T D}$ & $\%_{I T D}$ & $N_{I T D}$ & $\%_{I T D}$ & $N_{I T D}$ & $\%_{I T D}$ & $N_{I T D}$ & $\%_{I T D}$ \\
\hline$-3.5 \leq T<-0.5$ & 26 & 30.6 & 7 & 23.3 & 15 & 55.6 & 4 & 22.2 \\
$-0.5 \leq T<2.5$ & 17 & 21.0 & 2 & 6.1 & 9 & 37.5 & 6 & 27.3 \\
$2.5 \leq T<5.5$ & 43 & 23.4 & 11 & 20.4 & 18 & 37.5 & 14 & 18.2 \\
$5.5 \leq T<9.5$ & 9 & 14.8 & 1 & 7.7 & 4 & 18.2 & 4 & 15.4 \\
Total & 95 & 23.1 & 21 & 16.2 & 46 & 38.0 & 28 & 19.6 \\
\hline
\end{tabular}

Note that there are 17 galaxies which have no bar class specified in the RC3. Three of those $17(17.7 \%)$ are ITD's. 


\section{Conclusions}

The major conclusions we have drawn from this study are:

- The global existence rate of ITD's (all T-Types, all bar classes) in our sample of 411 galaxies is about $25 \%$

- There is no apparent correlation of ITD's with T-Type in this sample of objects

- Barred galaxies are more likely (by a factor of two or more) to be ITD than non-barred galaxies

- We predict that approximately $40 \%$ of optically non-barred galaxies may exhibit bars in the near IR

The principal question we are addressing in this paper is: Is the presence of a bar a necessary and/or sufficient condition for having an ITD? The presence of a bar is definitely not a sufficient condition for having an ITD, as only $38 \%$ of the barred galaxies in our sample are ITD's. Hence, the presence of a bar does not guarantee the presence of an ITD.

It is possible that bars are necessary for having an ITD, as only $16 \%$ of the non-barred galaxies exhibit an inner truncation. It is well-known that optically non-barred galaxies sometimes have bars when examined in the near-IR; our finding $16 \%$ of non-barred galaxies being ITD's coupled with the $38 \%$ rate of barred galaxies being ITD's suggests that perhaps $40 \%$ of all optically nonbarred galaxies have such IR bars. This is consistent with the observations of Pompea \& Rieke (1990), who find that at most $7 / 15(47 \%)$ and at least $4 / 15$ $(27 \%)$ of optically non-barred galaxies have bars in the near-IR.

Acknowledgments. W. B. was partially supported by STScI under contract NAS5-26555 for this work.

\section{References}

Baggett, W. E., Baggett, S. M., \& Anderson, K. S. J. 1992, BAAS, 24, 804

Baggett, W. E., Baggett, S. M., Anderson, K. S. J., \& Hamabe, M. 1993, BAAS, 24,1223

Freeman, K. C. 1970, ApJ, 160, 811

Jafarov, A. R. \& Zasov, A. V. 1989, Astr. Tsirk., No. 1540, 1

Kodaira, K., Okamura, S., \& Ichikawa, S. 1990, Photometric Atlas of Northern Bright Galaxies, University of Tokyo Press: Tokyo

Kormendy, J. 1977, ApJ, 217, 406

Ohta, K., Hamabe, M., \& Wakamatsu, K. 1990, ApJ, 357, 71

Pompea, S. M. \& Rieke, G. H. 1990, ApJ, 356, 416

de Vaucouleurs, G., de Vaucouleurs, A., Corwin, H. G., Buta, R. J., Paturel, G.

\& Fouque, P. 1991, Third Reference Catalogue of Bright Galaxies New

York: Springer-Verlag

de Vaucouleurs, G. 1953, MNRAS, 113, 134 